\title{
An Adaptive FAST Corner Detector based on High Contrast Grid Image
}

\author{
Teng Wu*, Zhili Zhang, Junyang Zhao, Fenggang Liu \\ Xian Research of High Technology, Xian, 710025, China \\ ${ }^{*}$ Corresponding author: Teng Wu
}

Keywords: image; high contrast enhancement; corner detection; adaptive threshold; entropy

\begin{abstract}
In this paper, an improved adaptive threshold algorithm was propose for grid image blocks with preprocessing in order to settle some defects as feature clustering, threshold fixation and noise sensitivity in FAST corner detection algorithms. Firstly, a high contrast enhancement filter algorithm is used to preprocess the image to suppress effects of the noise and enhance target characteristics. Then, based on the statistical analysis of image entropy and variance, a prediction equation for capacity of corner is proposed to adjust the threshold of the image block after the base threshold is determined to overcome the problem of image feature clustering. The experiments show that the adaptive thresholds of the improved algorithm are reasonable and the distribution of corner detection results is uniform, and the effect of noise and fuzzy images on the stability of the algorithm is well solved.
\end{abstract}

\section{Introduction}

Vehicle vision odometer system usually take feature-based image matching strategy in order to ensure real-time requirements. The features of images include distinctive features such as edge, corner, and circle and ellipse center. The corner feature is the point with high curvature on the edge or intersection of two and more edges ${ }^{[1-3]}$. Corner features can reflect the image gray-scale information and geometry information, which can effectively reduce the amount of calculation of image processing, and thus play an important role in vehicle vision positioning.

Image corner detection algorithms are generally divided into two categories: based on image blob feature and based on image gray-scale information. Methods based on BLOB feature such as SIFT algorithm ${ }^{[4]}$, SURF algorithm ${ }^{[5]}$ and CensurE algorithm ${ }^{[6]}$, the extracted features of which have strong significance, and rotation, affine, scale invariance. This kind of method often needs to construct the scale space to carry on the extreme value examination, causes the computation to be complex and the timeliness to be bad, so it is not suitable for the middle and high speed carrier platform. Methods based on the image gray information such as Harris algorithm, SUSAN algorithm and FAST algorithm ${ }^{[7]}$ have high detection efficiency, and are more suitable for the visual system with higher real-time requirements. The FAST corner detection algorithm proposed by Rosten et al. in 2010 can process PAL video images at less than $5 \%$ of available time, and the computational efficiency can meet the demands of real-time visual odometer applications. Although the algorithm own high operation efficiency, but in the application process, the image noise and edge burr sensitivity, while the extraction depends on the selection of corner threshold, and easily lead to the phenomenon of feature aggregation.

Based on the FAST algorithm, a high contrast enhancement filter is introduced to smooth the image, which can highlight the edge and corner with high contrast characteristic, and suppress the influence of noise. A new algorithm based on the image entropy and variance statistics is proposed to solve the problem of feature aggregation and threshold selection. Finally, the Hessian matrix and characteristic angle of supported domain are used to remove the non-corner. Experiments show that the results of improve algorithm have good distribution and robustness when it is used for corner extraction.

\section{Improved Threshold Adaptive FAST Corner Detection}

Based on the original FAST algorithm, this paper proposes a threshold adaptive FAST algorithm relying on the image high contrast enhancement filter preprocessing and adopting the grid image 
blocks method for the corner extraction(High Contrast -Adaptive- FAST, HA- FAST).

\subsection{Image Preprocessing by High Contrast Enhancement Filtering}

In order to eliminate the influence of image noise on the FAST algorithm and enhance the contrast between the foreground object and the background region, a high contrast enhancement filtering algorithm is introduced to preprocess the image ${ }^{[8]}$. The basic idea is to superimpose the smooth image and the high contrast features. The high contrast features of the image usually cluster on the edge region and the corners, so the image can get well smoothed by this filtering to eliminate noise and edge burr and the contrast between the corners and the background areas get Enhanced.

The high contrast enhancement filter for the image is:

$$
I(x, y)=f(x, y) \otimes h_{1}(x, y)+k\left[f(x, y) \otimes h_{2}(x, y)-f(x, y) \otimes h_{1}(x, y)\right] .
$$

In the formula, $f, I, h_{1}, h_{2}$ represent respectively for the original image, filtered image and filter template of different intensity, $k$ is the coefficient, generally taking 5 to 7 when dealing with images from moving vehicles.

The same image was smoothed, high-contrast extracted and high-contrast enhanced filtered, and results are shown in Figure 1. It is clear that gray-level mutations areas in the original image such as buildings, vehicles are obtained reservation and the edge noise is smoothed in high contrast enhancement images; and the relative grayscale contrast of smooth texture areas in the original image, such as roads, shadows, etc., is enhanced obviously, which increases the obvious features available for the image.

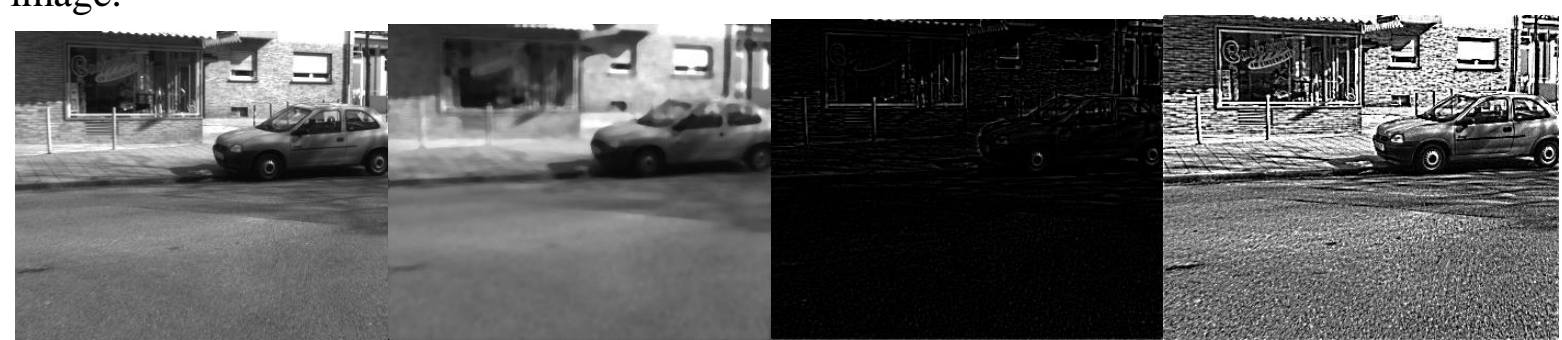

(a)The original image (b) The smoothed image (c) High contrast of the image (d) High contrast enhanced image

Fig.1 Images processed by different filtering methods
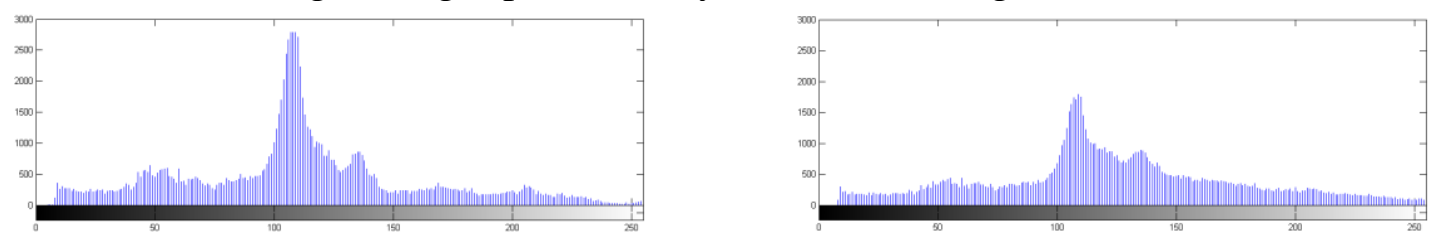

(a)Grayscale histogram of original image (b) Grayscale histogram of high contrast enhancement image

Fig. 2 Grayscale histogram of the original image and the filtered image

Calculate the grayscale histogram of the original image and the filtered image respectively, as shown in Figure 32 It can be seen that the peak of the grayscale distribution of the processed image is reduced, and the high grayscale and low grayscale parts are enhanced without damaging the whole image characteristics.

\subsection{Content of Corners Predicted by Image Entropy and Variance}

The image processed by high contrast enhancement filtering generally highlights the following information: roads, buildings, trees, road signs, vehicles and pedestrians. The gray-level variation and feature information of different target areas in the image are different. In this paper, we choose entropy and variance, which are the two features that can reflect image contrast and information capacity, to represent the corners density of gridding image block ${ }^{[9]}$.

Image entropy reflects the degree of image clutter, also describes the average amount of information of the image, expressed as the bit average of the image gray level set:

$$
H=-\sum_{i=0}^{255} P_{i} \ln P_{i} .
$$


In the formula, $p_{i}$ represent for the frequency at which the pixel with grayscale $i$ appears in the region. It can be seen from the above equation that the image entropy value is large when the image gray level is evenly distributed, and the image entropy value is small when the gray level of the image is gathered in partial gray level ${ }^{[10]}$.

The image variance reflects the grayscale contrast of the image, that is, the discrete degree of the grayscale distribution:

$$
V=\sum_{(x, y) \in s}(f(x, y)-\bar{f})^{2} .
$$

In the formula, $f, \bar{f}$ represent respectively for pixel grayscale and average grayscale. When the image grayscale contrast is high, the image variance is large; when the image grayscale contrast is low, the image variance is small.

Firstly, an image was segmented into 16 image blocks by gridding. The statistical properties of the image blocks are more obvious in entropy, variance and image classification than that of whole image, as shown in Fig 3.

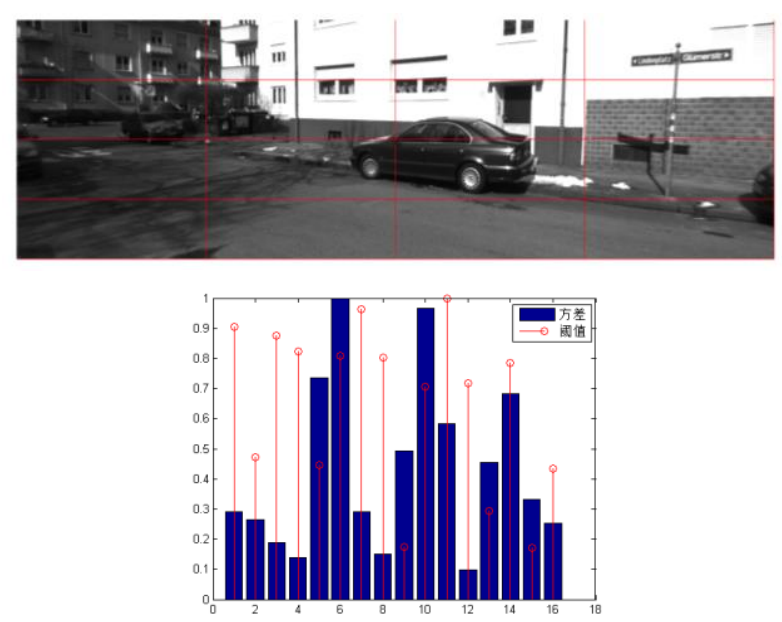

Fig.3 Image statistical properties of entropy and variance

The entropy and the variance information of each image block were calculated. The variance and entropy of the image correspond roughly to the image type: 1) The entropy is high, the variance is high, the grayscale variation of the corresponding image is intense; 2) The entropy is high, the variance is low, and the corresponding image has gray-similarity texture features; 3) The entropy is low, the variance is high, and the corresponding image is edge-clustering area with obvious grayscale contrast; 4) The entropy is low, the variance is low, which corresponds to the smoothed region where the gray level of the image is similar.

Then use the entropy and the variance as the function of the number of corners of the image block to select the grayscale threshold of FAST corner detection algorithm. The data concluding entropy $H$, variance $V$ and content of corner $C o C$ were extracted from the LIBVISO image set as training samples. The quadratic polynomial fitting was used to obtain the prediction equation:

$$
C o C=\left[\begin{array}{llllll}
V H & H & H & V^{2} & H^{2} & 1
\end{array}\right]\left[\begin{array}{c}
-1.1781 \\
-2.747 \\
0.009 \\
0.1469 \\
-0.684 \\
42.47
\end{array}\right] \text {. }
$$

The R-square of above formula is 0.88 , and the F-test has a high significance level, which can be accepted as the prediction equation. The fitting image is shown in Fig 4. 


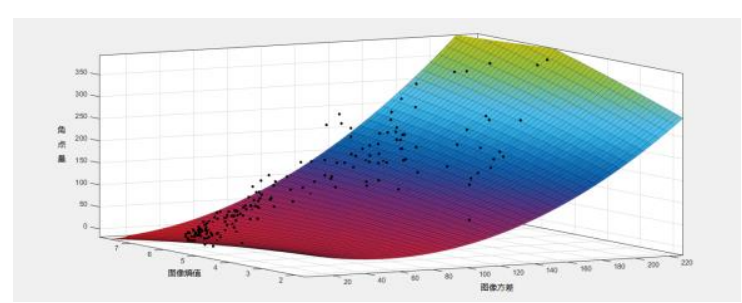

Fig.4 the entropy and variance fitted to the number of corners

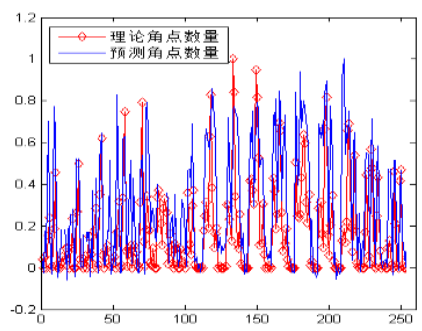

Fig. 5 Comparison of original data and fitting results

As shown in Fig. 5, the number of predicted corners and the number of original corners have the same trend approximately. However, the predicted values in some areas are obviously larger than the true values. These regions usually contain more edge texture and large information content, but the edges change smoothly and lack significant corner features.

\subsection{Adaptive Local Threshold Corner Detection}

If the same corner detection threshold is used in the detection of road images, it is easy for FAST algorithm to cause the detection results clustered on the trees and vehicle areas where the grayscale change is severe and the contour information is complex. Using the $4 \times 4$ grid to divide an image into 16 image blocks, and the strategy of adaptive local threshold for each image block can effectively solve the problem of feature aggregation and threshold selection.

1)Basic threshold determined by OTSU

OTSU is considered as the basic threshold of the whole image ${ }^{[11]}$ because in the high contrast enhanced image, the target grayscale and the background grayscale have the big difference.

The basic principle of the OTSU method is to obtain a grayscale $L$ that can divide the image into two parts with the largest inter-class variance, in order to distinguish between interests in the image of the target and not interests of the background:

$$
T=\operatorname{Arg} \max \left(\frac{\lambda}{\lambda+1}\right) \text {. }
$$

In the formula, $\lambda$ is a representation of the variance between classes?

$$
\lambda=\frac{\sigma_{O B}{ }^{2}(t)}{\sigma_{W}{ }^{2}(t)} \text {. }
$$

$\sigma_{O B}{ }^{2}(t), \sigma_{W}{ }^{2}(t)$ represent for intra-class and intra-class variance.

The grayscale $L$ determined by the OTSU is taken as the maximum threshold of image corner detection, and then the local threshold is adjusted according to the statistic characteristics of image block.

2)Local threshold $T h$ adjusted by $\mathrm{CoC}$

From equation (5), $\mathrm{CoC}$ can reflect the information amount of the image block and the grayscale variation, so It is reasonable to select the lower corner detection threshold for the image block with small $\mathrm{CoC}$ and the higher corner detection threshold for the image block with larger $\mathrm{CoC}$. Considering avoiding the excessive decrease of the threshold in the corners information-rich region, and effectively improving the corner extraction result of the small $C o C$ region, we define the adaptive threshold function as:

$$
T h=T \sqrt{\operatorname{CoC}_{i, j} / \max \operatorname{CoC}} \text {. }
$$

Th Can adaptively adjust its value according to the content of corners in the image block so that 
each block in the whole image can get a certain number of significant corners to reduce the error caused by the camera imaging accuracy. At the same time, the method is simple in calculation process and avoids the iterative operation, which has no significant effect on the efficiency of the algorithm.

\section{Experiments and Analysis}

In this paper, the road images from LIBVISO are taken as the experimental subjects. A series of contrast experiments are designed based on the Harris algorithm, the original FAST algorithm and HA-FAST algorithm. The number, the dispersion, the time-consuming and the algorithm stability are calculated from the corners respectively to verify the effectiveness of HA-FAST algorithm proposed above.

Comparison of different corner detection methods. Harris algorithm, the original FAST algorithm and HA-FAST algorithm were used to detect the corners of the same mage, and the test results are shown in Figure 8.

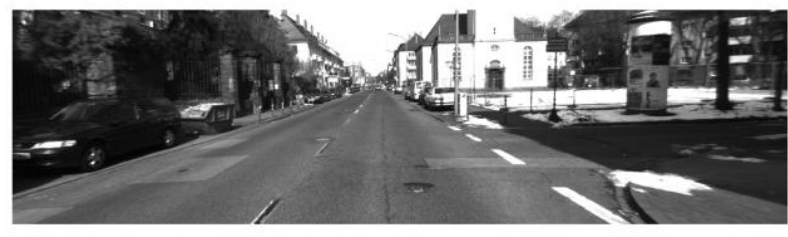

(a)The original image

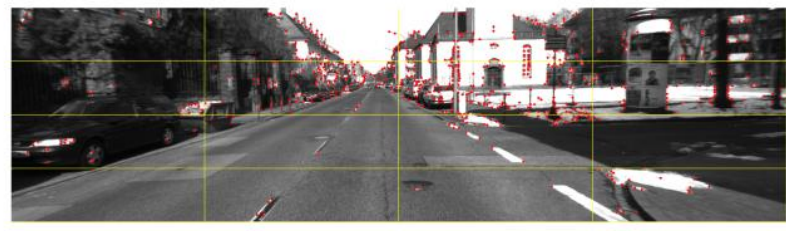

(b)Harris algorithm corner detection results

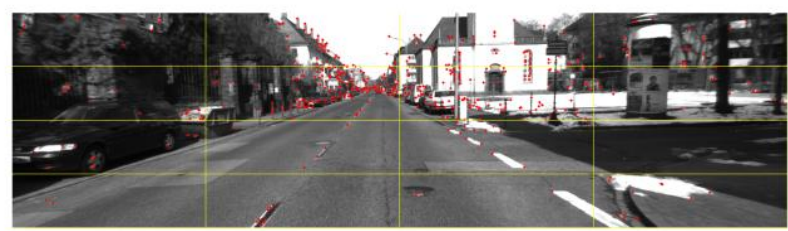

(c)The original FAST algorithm corner detection results

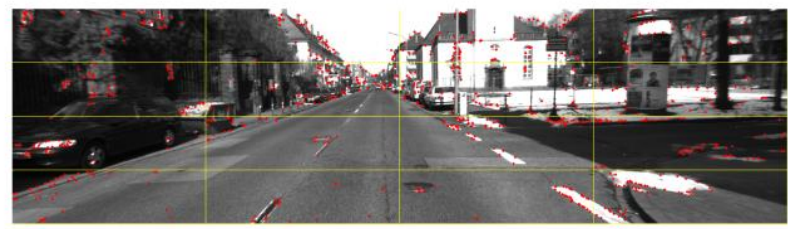

(d)HA-FAST algorithm corner detection results Fig.6 Comparison of Different Corner Detection

The original image is the grayscale of the road scene captured form moving vehicles. The image contains scenes such as roads, buildings, vehicles, trees and road signs. The detection results of Harris algorithm are shown in Fig.6 (b). The algorithm is more accurate in corner location than other algorithms, but the detection process takes longer time and fewer corners are detected. The detected corners are obviously concentrated in same certain areas and some corners of the low contrast is missed. The original FAST algorithm although takes short time, the detection results as Fig. 6 (c) show that a large number of features are clustered in the region $(2,2)$, and in the regions with low grayscale variation $(3,2),(4,1),(4,3)$ the number of detected corners is relatively small. And some significant corners of the image such as building edge vertex, landmark endpoint and so on are missed. The detection results of HA-FAST algorithm is shown in Fig. 6 (d), it can be seen that the low-contrast 
corners in the regions with low grayscale variation, especially in the road and shadow areas and in texture region can be detected. Each block of the grid image has a certain number of detected corners, and the corners are evenly distributed throughout the image.

Define the aggregation of corners:

$$
C=\frac{N_{\max }-N_{\min }}{\bar{N}} \text {. }
$$

In the formula, $N_{i}$ is the number of corners of each grid image block? The larger $C$ is, the higher the degree of corners aggregation, and the worse the feature distribution. The experimental data are shown in Table 1.

Table 1 Performance parameter table of different corner detector

\begin{tabular}{cccc}
\hline Algorithm & Number of corners N & Aggregation of corners C & Time consuming \\
\hline Harris & 696 & 4.46 & 5.44 \\
FAST & 464 & 5.10 & 0.96 \\
HA-FAST & 852 & 0.97 & 1.53 \\
\hline
\end{tabular}

From the experimental results, the HA-FAST algorithm can effectively avoid the problem of corners clustering and reduce the false detection rate of the significant corners. The improved algorithm also has the blur image and noise image tolerance which the original FAST algorithm does not have, and the whole robustness of the algorithm is strong.

\section{Conclusion}

In this paper, an HA-FAST corner detection algorithm is proposed. The image is preprocessed by high contrast image enhancement technique to suppress the noise and enhance the image feature area. The content of image corners is constructed by quantitative analysis of image entropy and variance information. Based on this index grid image adaptive threshold for corner detection is calculated. Experimental results show that the algorithm has good corner detection effect, and the algorithm is robust. But the algorithm is not enough to analyze the statistic characteristic of the image. The formula of the corner-point description is only for the image captured by vehicle camera, and the method generality has to be improved.

\section{References}

[1]. Sonka M, Hlavac V, Ceng R B D M. Image Processing, Analysis, and Machine Vision [M]. Thomson Learning, 2008: 464-466.

[2]. Zhang G J. Machine Vision [M]. Beijing: Science Press, 2005: 50-51.

[3]. Zhao P. Machine vision theory and application [M]. Beijing: Publishing House of Electronics Industry, 2011: 43-44.

[4]. D.Lowe. Distinctive Image Features from Scale-invariant Key points [J], International Journal of Computer Vision, 2004, 60(2): 91-110.

[5]. Bay H. Tuytelaars T. Gool L.V.SURF: Speeded up Robust Features [J]. Computer Vision 2006: 404-417.

[6]. M. Agrawal, K. Konolige, M. R. Blas. CenSurE: Center Surround Extremas for Realtime Feature Detection and Matching [C]. In Proceedings of 10th European Conference on Computer Vision (ECCV), 2008.

[7]. E. Rosten, R. Porter, T. Drummond. Fast and Better: A Machine Learning Approach to Corner Detection [C]. IEEE Transactions on Pattern Analysis and Machine Intelligence, 2010. 32(1): 105-119.

[8]. Choudhury P, Tumblin J. The Trilateral Filter for High Contrast Images and Meshes.[C]// Eurographics Workshop on Rendering Techniques, Leuven, Belgium, June. 2003:186-196.

[9]. Porway J, Wang K, Yao B, et al. A hierarchical and contextual model for aerial image understanding[C]// IEEE Conference on Computer Vision \& Pattern Recognition. 2008:1-8. 
[10]. Chen Y F, Wu Q D, Zhao W D, et al. Fast chan-vese segmentation algorithm based on image entropy[J]. Journal of Tongji University. Natural Science, 2011, 39(5): 738-744.

[11]. Vala M H J, Baxi A. A review on Otsu image segmentation algorithm [J]. International Journal of Advanced Research in Computer Engineering \& Technology, 2013, 2(2).

[12]. Lakemond R, Fookes C, Sridharan S. Affine Adaptation of Local Image Features Using the Hessian Matrix[C]// IEEE International Conference on Advanced Video and Signal Based Surveillance. IEEE, 2009:496-501.

[13]. WANG H Z, WU F C, WANG X G. Corner detection and sub-pixel localization based on local orientation distribution [J]. Journal of Software, 2008, 11: 019. 